\title{
NEURAL BLIND SEPARATION OF COMPLEX SOURCES BY EXTENDED APEX ALGORITHM (EAPEX)
}

\author{
Simone Fiori, Aurelio Uncini, and Francesco Piazza
}

Dept. Electronics and Automatics - University of Ancona (Italy)

E-mail:simone@eealab.unian.it

\begin{abstract}
Blind Source Separation by non-classical (non-quadratic) neural Principal Component Analysis has been investigated by several papers over the recent years, even if particular attention has been paid to the real-valued sources case. The aim of this work is to present an extension of the KungDiamantaras' APEX learning rule to non-quadratic complex optimization, and to show the new approach allows blind separation of complex-valued source signals from their linear mixtures.
\end{abstract}

\section{INTRODUCTION}

Blind Source Separation (BSS) by Independent Component Analysis (ICA) of complex-valued data $[2,4,10]$ is a meaningful problem that has been investigated in a very few papers, while much more attention has been paid to develop several different algorithms for performing BSS-ICA of realvalued data. Among others, those methods based on nonlinear extensions of Principal Component Analysis (PCA) have raised much interest in the Neural Network community (see for example $[6,9]$ and references therein). It has been proven by many papers that adding non-linearity to linear PCA neural networks makes them able to improve the independence of their outputs so as to allow blind separation of independent sources [6,9]. Recently, some attempts have been made in order to extend the best known PCA algorithms to the complex case. In [1] Chen and Hou presented an heuristic complex version of the well-known APEX algorithm [5], in [3] De Castro et al. gave an heuristic complex extension of the GHA algorithm [11] successively generalized to the non-linear case by Fiori and Piazza [8], while in [7] Fiori and Uncini proposed a formal derivation of a large class of complex PCA neural algorithms containing, as a special case, the one found in [1].

In this paper we formally derive a new learning algorithm as a non-linear complex generalization of APEX, and discuss the choice of the non-linearity under the theoretical framework proposed by Sudjianto and Hassoun [12] ex-

This research was supported by the Italian MURST. tended to the complex case. Then we show how a particular non-linearity, called Rayleigh function, allows the neural network to separate out mixed independent complex-valued source signals.

\section{EXTENDED APEX ALGORITHM (EAPEX)}

Consider the complex-weighted neural network with input $\mathbf{x} \in \mathcal{C}^{p}$ and output $\mathbf{y} \in \mathcal{C}^{m}$, with $m \leq p$, described by the following relationships:

$$
z_{k}=\mathbf{w}_{k}^{\#} \mathbf{x}, y_{k}=z_{k}+\mathbf{h}_{k}^{\#} \mathbf{y},
$$

where $\mathbf{z} \in \mathcal{C}^{m}$, vectors $\mathbf{w}_{k} \in \mathcal{C}^{p}$ represent the network's direct connections, vectors $\mathbf{h}_{k} \in \mathcal{C}^{p}$ represent the network's lateral connections, and superscript \# denotes conjugate transpose. In the following $E_{\mathbf{x}}[\cdot]$ denote mathematical expectation with respect to $\mathbf{x}$.

In [7] it has been shown that it is possible to define a pair $(J, C)$ of objective functions as follows:

$$
\begin{aligned}
& J\left(\mathbf{w}_{k}\right) \stackrel{\text { def }}{=} \sum_{k=1}^{m} P_{k}\left(\mathbf{w}_{k}\right)+\frac{1}{2} \sum_{k=1}^{m} \lambda_{k}\left(1-\mathbf{w}_{k}^{\#} \mathbf{w}_{k}\right), \\
& C\left(\mathbf{h}_{k}\right) \stackrel{\text { def }}{=} \frac{1}{2} \sum_{k=1}^{m} E_{\mathbf{x}}\left[\left|y_{k}\right|^{2}\right]+\sum_{k=1}^{m} E_{\mathbf{x}}\left[\psi_{k}\right]\left(\mathbf{h}_{k}^{\#} \mathbf{h}_{k}\right),
\end{aligned}
$$

where $P_{k}=E_{\mathbf{x}}\left[\left|y_{k}\right|^{2}\right]$ and $\lambda_{k}$ and $E_{\mathbf{x}}\left[\psi_{k}\right]$ are Lagrange multipliers, such that maximizing $J$ with respect to each $\mathbf{w}_{k}$ only and simultaneously minimizing $C$ with respect to each $\mathbf{h}_{k}$ only, gives a set of learning equations allowing the network to perform Principal Component Analysis of incoming complex-valued data.

In this paper we generalize the criterion $J\left(\mathbf{w}_{k}\right)$ by defining instead:

$$
P_{k} \stackrel{\text { def }}{=} E_{\mathbf{x}}\left[f\left(\left|y_{k}\right|\right)\right],
$$

where $f(u): \mathcal{R}_{0}^{+} \rightarrow \mathcal{R}_{0}^{+}$is a function continuously differentiable almost everywhere, non-decreasing with a unique minimum in $u=0$. In this case we thus have:

$$
\frac{\partial J\left(\mathbf{w}_{k}\right)}{\partial \mathbf{w}_{k}}=E_{\mathbf{x}}\left[f^{\prime}\left(\left|y_{k}\right|\right) \frac{\partial\left|y_{k}\right|}{\partial \mathbf{w}_{k}}\right]
$$


while from [7] we know that:

$$
\frac{\partial\left|y_{k}\right|^{2}}{\partial \mathbf{w}_{k}}=2 \mathbf{x} y_{k}^{\star}=2\left|y_{k}\right| \frac{\partial\left|y_{k}\right|}{\partial \mathbf{w}_{k}},
$$

where superscript ${ }^{\star}$ denotes conjugation. Moreover, from [7] we also know that:

$$
\frac{\partial\left|y_{k}\right|^{2}}{\partial \mathbf{h}_{k}}=2 \mathbf{y}_{[k]} y_{k}^{\star},
$$

where $\mathbf{y}_{[k]} \stackrel{\text { def }}{=}\left[\begin{array}{lllllll}y_{1} & y_{2} & \cdots & y_{k-1} & 0 & \cdots & 0\end{array}\right]^{T}$ with $k>1$, and $\mathbf{y}_{[1]} \stackrel{\text { def }}{=}\left[\begin{array}{lllll}0 & 0 & \cdots & 0 & 0\end{array}\right]^{T}$. Define now $F(u) \stackrel{\text { def }}{=} \frac{f^{\prime}(u)}{u}$; then we may write:

$$
\begin{aligned}
& \frac{\partial J\left(\mathbf{w}_{k}\right)}{\partial \mathbf{w}_{k}}=E_{\mathbf{x}}\left[F\left(\left|y_{k}\right|\right) y_{k}^{\star} \mathbf{x}\right]-\lambda_{k} \mathbf{w}_{k}, \\
& \frac{\partial C\left(\mathbf{h}_{k}\right)}{\partial \mathbf{h}_{k}}=E_{\mathbf{x}}\left[y_{k}^{\star} \mathbf{y}_{[k]}\right]+E_{\mathbf{x}}\left[\psi_{k}\right] \mathbf{h}_{k} .
\end{aligned}
$$

From optimization theory we know the optimal multipliers $\lambda_{k}$ can be found by solving the following equations:

$$
\left.\mathbf{w}_{k}^{\#} \frac{\partial J}{\partial \mathbf{w}_{k}}=E_{\mathbf{x}}\left[F\left(\left|y_{k}\right|\right)\right] y_{k}^{\star} z_{k}\right]-\lambda_{k}=0,
$$

whose solutions give the optimal gradients:

$$
\left(\frac{\partial J\left(\mathbf{w}_{k}\right)}{\partial \mathbf{w}_{k}}\right)^{\circ \mathrm{pt}}=E_{\mathbf{x}}\left[F\left(\left|y_{k}\right|\right) y_{k}^{\star}\left(\mathbf{x}-z_{k} \mathbf{w}_{k}\right)\right] .
$$

Standard Kuhn-Tucker theory shows that it is not possible to find optimal multipliers $E_{\mathbf{x}}\left[\psi_{k}\right]$, instead. Then, by letting weight vectors adapt by means of gradient steepest ascent/descent algorithms, we find the new learning rule:

$$
\begin{aligned}
& \Delta \mathbf{w}_{k}=\eta \frac{\partial J}{\partial \mathbf{w}_{k}}=\eta E_{\mathbf{x}}\left[F\left(\left|y_{k}\right|\right) y_{k}^{\star}\left(\mathbf{x}-z_{k} \mathbf{w}_{k}\right)\right], \\
& \Delta \mathbf{h}_{k}=-\eta \frac{\partial C}{\partial \mathbf{h}_{k}}=-\eta E_{\mathbf{x}}\left[y_{k}^{\star} \mathbf{y}_{[k]}\right]-\eta E_{\mathbf{x}}\left[\psi_{k}\right] \mathbf{h}_{k} \\
& k=1,2, \ldots, m, \eta>0,
\end{aligned}
$$

that is referred to as Extended APEX Algorithm (EAPEX) in that for $f(u)=\frac{1}{2} u^{2}, \psi_{k}=\left|y_{k}\right|^{2}$, as long as $y_{k} \cong z_{k}$, and in presence of real valued data, it resembles the APEX learning algorithm by Kung and Diamantaras [5].

In the next Section we discuss a choice of $F(\cdot)$ arising from an interpretation of non-quadratic Hebbian learning due to Sudjianto and Hassoun [12] extended to the complex case.

\section{THE SUDJIANTO-HASSOUN INTERPRETATION OF NON-QUADRATIC HEBBIAN LEARNING}

In [12], Sudjianto and Hassoun considered the problem of maximizing a criterion $P(\mathbf{w}) \stackrel{\text { def }}{=} E\left[S^{2}\left(\mathbf{w}^{T} \mathbf{x}\right)\right]$ subject to the restriction $\mathbf{w}^{T} \mathbf{w}=1$, where $y=\mathbf{w}^{T} \mathbf{x}$ is the output of a single-unit real-weighted neural network and $S(\cdot)$ is a generic saturating sigmoidal function, for instance such that $S(\cdot) \in[-1,+1]$, that is the same problem addressed in Section 2 particularized to $m=1$. They noted that maximizing the variance of a saturating function of $y$ leads the neuron to prefer configurations $\mathbf{w}$ corresponding to values of $S(y)$ concentrated near the extremes -1 and +1 . If the quantity $z=S(y)$ is perceived as a new random variable with probability density function $q_{Z}(z \mid \mathbf{w})$, this makes U-shaped the distribution $q_{Z}$ [12]. The gradient steepest ascent learning rule for the neuron is:

$$
\frac{d \mathbf{w}}{d t}=\frac{\partial J}{\partial \mathbf{w}}=\left(\mathbf{I}-\mathbf{w}^{T}\right) E_{\mathbf{x}}[\ell(y) \mathbf{x}],
$$

where $\ell(u) \stackrel{\text { def }}{=} 2 S^{\prime}(u) S(u)$. Denote now by $q_{Y}(y \mid \overline{\mathbf{w}})$ the probability density function of the random variable $y$ due to a configuration $\overline{\mathbf{w}}$, and with $Q_{Y}(y \mid \overline{\mathbf{w}})$ its cumulative distribution function, namely:

$$
Q_{Y}(y \mid \overline{\mathbf{w}}) \stackrel{\text { def }}{=} \int_{-\infty}^{y} q_{Y}(\eta \mid \overline{\mathbf{w}}) d \eta .
$$

Assume then $S(y)=2 Q_{Y}(y \mid \overline{\mathbf{w}})-1$. In this case it is well known [12] that $z$ will be uniformly distributed within $[-1,+1]$. The central idea developed by Sudjianto and Hassoun is that the learning rule (9) will converge to a weight vector surely different from $\overline{\mathbf{w}}$, since the rule seeks a Ushaped distribution of $z$, that is, a distribution that deviates away from a uniform one. If, for instance, $\mathrm{x} \in \mathcal{R}^{2}, x_{1}$ has a pdf $q_{1}\left(x_{1}\right)$ and $x_{2}$ has a pdf $q_{2}\left(x_{2}\right)$ different from $q_{1}$, then choosing $S(y)=2 Q_{1}(y)-1$ makes the rule (9) able to filter the signal $x_{1}$ allowing for $y=x_{2}$ at convergence, and viceversa. In other words, the rule (9) behaves as a probabilistic filter.

Consider now the extension of the previous theory to the complex case. Define the cost function:

$$
P(\mathbf{w}) \stackrel{\text { def }}{=} E_{\mathbf{x}}\left[S^{2}(|y|)\right] .
$$

In our case we assume:

$$
S(|y|)=Q_{|Y|}(|y|) \stackrel{\text { def }}{=} \int_{0}^{|y|} q(\eta) d \eta,
$$

where $q(\cdot)$ represents a generic probability density function. Clearly this implies $f(u)=S^{2}(u)$, hence:

$$
f^{\prime}(u)=2 q(u) \int_{0}^{u} q(\eta) d \eta
$$

Ultimately it is clear that training each neuron of a linear complex-weighted neural network by means of the learning rule EAPEX with the non-linearity (11) causes the network to learn connection strengths that filter the outputs so that 
the probability density function of the output moduli $\left|y_{k}\right|$ deviates away from $q(\cdot)$. In the next section it will be made clear how this principle could be employed for separating out independent complex signals from their linear mixtures.

\section{APPLICATION TO BLIND COMPLEX SOURCE SEPARATION}

Suppose input $\mathbf{x}$ contains a complex linear mixture of statistically independent signals [10], and that one of these signals is a Gaussian noise of the form $v=r+i s$, where both $r$ and $s$ are zero-mean Gaussian random variables of variance $\sigma^{2}$. Then it is known that the modulus $|v|$ follows the Rayleigh distribution:

$$
q_{\mathrm{R}}(|v|)=\frac{|v|}{\sigma^{2}} \exp \left(-\frac{|v|^{2}}{2 \sigma^{2}}\right)
$$

Then by formula (11) we find:

$$
f_{\mathrm{R}}^{\prime}(u)=\frac{2 u}{\sigma^{2}}\left[\exp \left(-\frac{u^{2}}{2 \sigma^{2}}\right)-\exp \left(-\frac{u^{2}}{\sigma^{2}}\right)\right] \Gamma(u),
$$

where $\Gamma(u)$ is the unitary step. Figure 1 depicts the Rayleigh non-linearity $F_{\mathrm{R}}(u)=f_{\mathrm{R}}^{\prime}(u) / u$ for a unitary noise power. In this case it is possible to express the cumulative distribu-

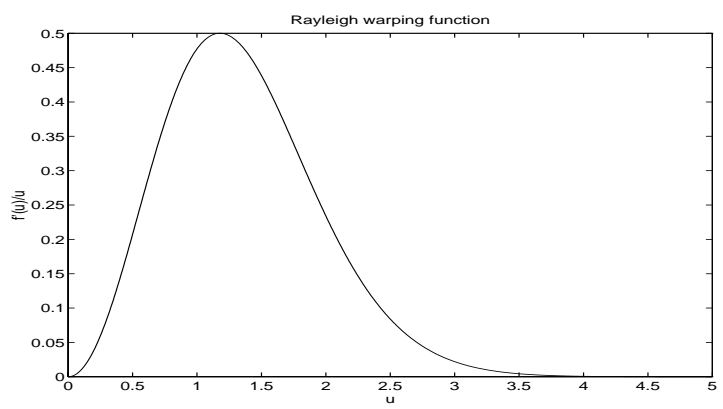

Figure 1: Rayleigh warping function for $\sigma^{2}=1$.

tion function in closed form simply as:

$$
Q_{\mathrm{R}}(u)=1-\exp \left(-\frac{u^{2}}{2 \sigma^{2}}\right) .
$$

By using in the EAPEX algorithm the function $F_{\mathrm{R}}(u)$ it is then possible to separate out independent complex-valued signals mixed by a unitary operator. The general problem where generic linear mixtures are concerned can be solved by pre-whitening the data $[2,10]$.

\section{EXPERIMENTAL RESULTS}

As a numerical example, suppose input $\mathrm{x} \in \mathcal{C}^{4}$ is formed by a linear mixture of four independent signals arranged in a vector $\mathbf{s} \in \mathcal{C}^{4}$. Signal $s_{1}$ is QAM4 and $s_{2}$ is QAM16, both with small Gaussian phase deviation; signal $s_{3}$ is PSK, and $s_{4}$ is a Gaussian noise of variance $\sigma^{2}=0.5$. The mixture is computed as $\mathbf{x}=\mathbf{M s}$, where $\mathbf{M}$ is a randomly generated $4 \times 4$ complex matrix. The first row of Figure 2 depicts the independent signals while second row shows the obtained four mixtures.
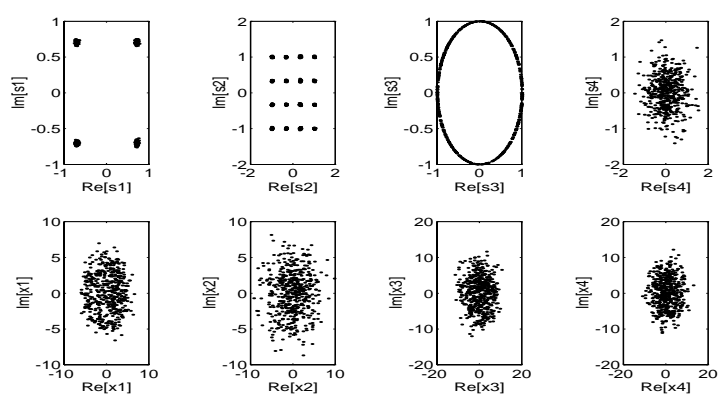

Figure 2: The four independent signals and the four mixtures of them.

By means of the Sudjianto-Hassoun principle, a linear neural network with four inputs and four outputs, trained by the learning rule EAPEX with the Rayleigh non-linearity, should be able to recover the independent signals except for a phase shift and a random permutation [2] after mixture prewhitening. Simulation results are shown in Figure 3: The first row depicts whitened mixtures obtained by means of the well-known Laheld-Cardoso's standardizing algorithm [10] in its stabilized version:

$$
\Delta \mathbf{U}=\gamma \frac{\mathbf{U}(\mathbf{I}-\mathbf{v v} \#)}{|1+\gamma \mathbf{v} \# \mathbf{v}|}, \mathbf{v}=\mathbf{U}^{\#} \mathbf{y},
$$

with $\gamma=0.0005$. The second row shows the last 100 outputs of the network trained by EAPEX on the prewhitened data $\mathbf{v}$, with the choice $\psi_{k} \stackrel{\text { def }}{=}\left|y_{k}\right|$ (for a discussion on the possible choices of the $\psi_{k}$ 's see [7]), $\eta=0.005, \sigma^{2}=0.5$ and $\mathbf{W}(0)=$ I. Figure 4 shows the histograms of the
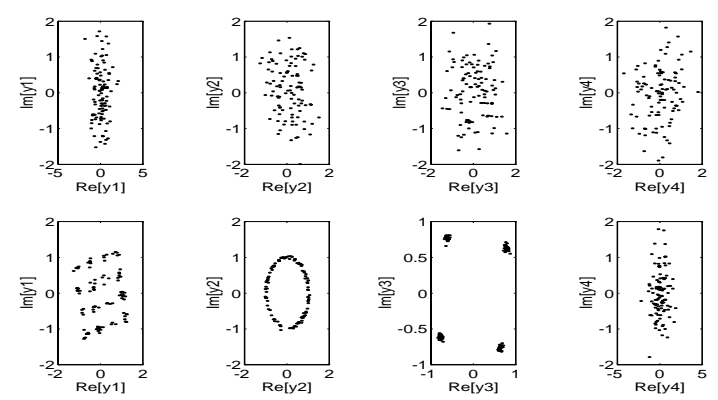

Figure 3: Network's output after learning by rule EAPEX. 

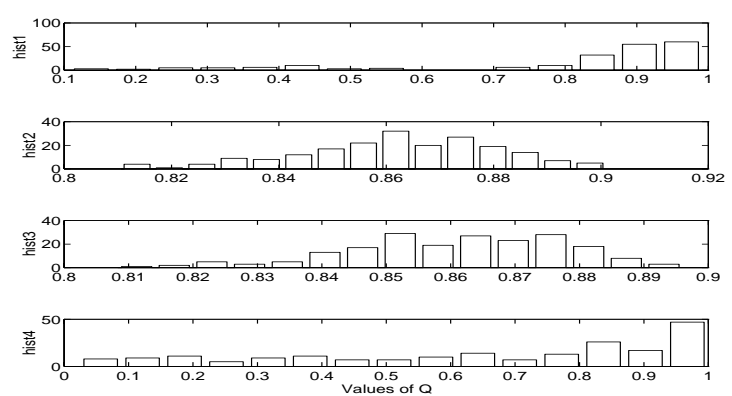

Figure 4: Functions $Q_{\mathrm{R}}(\cdot)$ histograms.

last 200 samples of $Q_{\mathrm{R}}\left(\left|y_{1}\right|\right), \ldots, Q_{\mathrm{R}}\left(\left|y_{4}\right|\right)$, while Figure 5 depicts the residual interference defined on the basis of the separation product $\Pi \stackrel{\text { def }}{=} \mathbf{W}^{\#} \mathbf{U} \mathbf{U}^{\#} \mathbf{M}$ as the sum of the 12 smallest numbers among $\left|\Pi_{i j}\right|^{2}$ divided by the sum of the 4 largest numbers among $\left|\Pi_{i j}\right|^{2}$.

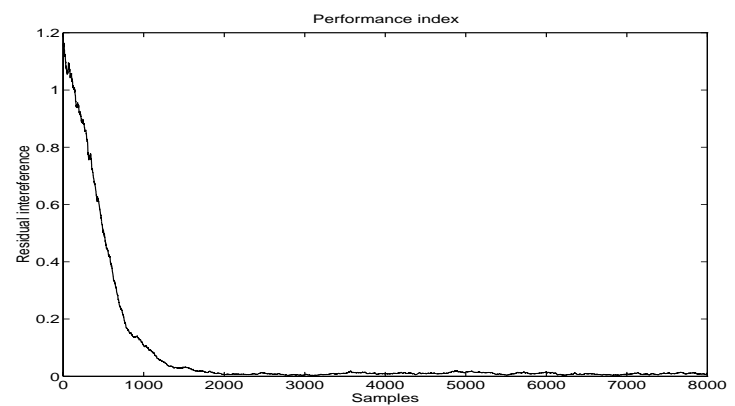

Figure 5: Residual intereference.

Simulation results show that the network is able to recover the independent signals. The histograms $Q_{\mathrm{R}}\left(\left|y_{1}\right|\right)$, $Q_{\mathrm{R}}\left(\left|y_{2}\right|\right)$ and $Q_{\mathrm{R}}\left(\left|y_{3}\right|\right)$ are in good accordance with the signals in Figure 3, while the presence of a peak in +1 on the histogram of $Q_{\mathrm{R}}\left(\left|y_{4}\right|\right)$ confirms that the fourth neuron cannot separate out the Gaussian noise and its output contains a mixture of the other source signals, as expected.

\section{CONCLUSION}

Blind separation by extended Hebbian algorithm has become a fruitful research field, as already pointed out also by Hyvärinen and Oja [6] who have recently proposed an algorithm for blind separation of real-valued signals from orthogonal mixtures via linear networks based on non-classical Hebbian learning. In this paper a new adapting rule for linear neural networks as generalization of APEX learning has been presented by using some results of an our previous work [7]. It provides a generalization in that it applies to complex-weighted neural networks and embeds non-linearity in the classical Hebbian learning. A particular choice of the non-linearity is discussed by recalling the SudjiantoHassoun interpretation of non-classical Hebbian learning extended to the complex case.

\section{REFERENCES}

[1] Y. CHEN AND C. Hou, High resolution adaptive bearing estimation using a complex-weighted Neural Network, Proc. of IEEE-ICASSP, Vol. II, pp. 317 320, 1992

[2] P. Comon, Independent Component Analysis, a new concept ?, Signal Processing, Vol. 36, pp. 287 - 314, 1994

[3] M.C.F. De Castro, F.C.C. De Castro, J.N. Amaral, And P.R.G. Franco, A Complex Valued Hebbian Learning Algorithm, Proc. of IEEE-ICNN, pp. $1235-1238,1998$

[4] G. Desodt And D. Muller, Complex ICA applied to the separation of radar signals, Proc. of EUSIPCO, Vol. I, pp. 665 - 668, 1990

[5] K.I. Diamantaras And S.-Y. Kung, Principal Component Neural Networks: Theory and Applications, J. Wiley \& Sons, 1996

[6] A. HyVÄRINEN AND E. OJA, Independent Component Analysis by general non-linear Hebbian-like rules, Signal Processing, Vol. 64, No. 3, pp. 301 - 313, 1998

[7] S. Fiori AND A. UnCINI, A unified approach to laterally-connected neural networks, Proc. EUSIPCO, Vol. I, pp. 379 - 382, 1998

[8] S. FIORI AND F. PIAZZA, Neural blind separation of complex sources by extended Hebbian learning (EGHA), These proceedings

[9] J. KARHUnen And J. Joutsensalo, Representation and separation of signals using nonlinear PCA type learning, Neural Networks, Vol. 7, No. 1, pp. 113 $-127,1994$

[10] B. LAHELD AND J.F. CARDoso, Adaptive source separation with uniform performance, Proc. of EUSIPCO, Vol. 1, pp. 183 - 186, 1994

[11] T.D. SANGER, Optimal unsupervised learning in a single-layer linear feedforward neural network, Neural Networks, Vol. 2, pp. 459-473, 1989

[12] A. Sudjianto And M.H. Hassoun, Nonlinear Hebbian rule: A statistical interpretation, Proc. of IEEE-ICNN, Vol. 2, pp. 1247 - 1252, 1994 